\title{
Intoxicação infantil por chumbo: uma análise discriminante entre os fatores de risco e de proteção
}

\section{Childhood lead poisoning: A discriminant analysis between risk and protection}

\section{Los niños envenenados por plomo: Análisis discriminante entre el factores de riesgo y protección}

\author{
Denise Dascanio* \\ Universidade Paulista - UNIP, Bauru, São Paulo, Brasil
}

\section{Zilda Aparecida Pereira Del Prette**}

Universidade Federal de São Carlos - UFSCar, São Carlos, São Paulo, Brasil

\section{Anne Marie Germane Victorine Fontaine*** \\ Universidade do Porto - UP, Porto, Portugal}

\begin{abstract}
RESUMO
As habilidades sociais são reconhecidas como fator de proteção ao longo do desenvolvimento humano, contra os impactos dos fatores de risco. Neste estudo, considerando a situação de risco de crianças e adolescentes contaminadas por chumbo, buscou-se identificar as variáveis (habilidades sociais, práticas parentais, problemas de comportamento e competência acadêmica) que as diferenciam das não contaminadas e a possível função protetora das habilidades sociais sobre problemas de comportamento em ambos os grupos. Participaram desta pesquisa 100 crianças, suas mães e professores. Os problemas de comportamento, a competência acadêmica e as habilidades sociais das crianças foram avaliados pelo Sistema de Avaliação de Habilidades Sociais (SSRS-BR); as habilidades sociais dos adolescentes pelo Inventário de Habilidades Sociais para Adolescentes (IHSA-Del-Prette). As práticas parentais foram avaliadas pelo Inventário de Estilos Parentais (IEP). Com base nas análises de variância, discriminante e de mediação, foi possível identificar diferenças entre os dois grupos, com maior comprometimento nas crianças contaminadas, exceto para a habilidade social de assertividade autoavaliada, cujo resultado apontou maior repertório para as crianças intoxicadas por chumbo. Comprovou-se também a função mediadora das habilidades sociais minimizando o impacto da plumbemia sobre os problemas de comportamento. O conjunto dos resultados reforça a ideia de que um repertório elaborado de habilidades sociais pode estar relacionado com a competência cognitiva das crianças e adolescentes.
\end{abstract}

Palavras-chave: habilidades sociais, intoxicação por chumbo, fatores de proteção. 


\begin{abstract}
Social skills are recognized as protection factor during human development, against the impacts of risk factors. In this study, considering the risk of children and adolescents contaminated by lead, we sought to identify variables (social skills, parenting practices, behavior problems and academic competence) that distinguish them from uncontaminated and the possible protective role of social skills on behavioral problems in both groups. A sample of 100 children, their mothers and teachers participated in this study. Behavior problems and academic competence were assessed by Social Skills Rating (SSRS-BR); the social skills by SSRS-BR and Inventory of Social Skills for Adolescents (IHSA- Del Prette). Parenting practices were assessed by the Inventory of Parental Styles (IEP). Based on analyzis of variance, discriminant and mediation, it was possible to identify differences between the two groups, with greater impairment in children contaminated, except for the social skill self evaluation assertiveness, and the result showed the best repertoire for lead-poisoned children. It was proved also the mediating role of social skills while minimizing the impact of blood lead level on behavior problems. The overall results showed the notion that a repertoire of social skills may be related to cognitive skills of children and adolescents.
\end{abstract}

Keywords: social skills, blood lead level, protection factors.

\title{
RESUMEN
}

Las habilidades sociales se reconocen como factor de protección sobre el desarrollo humano, de los efectos de los factores de riesgo. En este estudio, teniendo en cuenta la situación de los niños y adolescentes intoxicados con plomo, hemos tratado de identificar las variables (habilidades sociales, prácticas parentales, problemas de conducta y de competencia académica) que las diferencian de no intoxicado y el posible papel protector de las habilidades sociales en los problemas de conducta en los dos grupos. El estudio reunió a 100 niños, sus madres y maestros. Los problemas de conducta y competencia académica se evaluaron mediante SSRS-BR; habilidades sociales por Sistema de evaluación de habilidades sociales (SSRS-BR) y Inventario de Habilidades Sociales Adolescentes (IHSA-DelPrette). Prácticas parentales fueron evaluados por lo Inventario de Estilos Parentales (IEP). Con base en el análisis de la varianza, discriminante y la mediación fue posible identificar las diferencias entre los dos grupos, con mayor impacto en los niños infectados, a excepción de la asertividad autoavaliada, cuyo resultado mostró mejor repertorio para niños intoxicados por plomo. Se demuestra también el papel mediador de las habilidades sociales, minimizando el impacto de plomo en la sangre en los problemas de la conducta. Los resultados globales refuerzan la nocíon de que un repertorio de habilidades sociales puede estar relacionado con las habilidades cognitivas de los niños y adolescentes.

Palabras clave: habilidades sociales, trastornos tóxicos, factor de protección.

\section{Introdução}

A exposição ao chumbo é um grave problema de saúde pública e, embora as concentrações de chumbo no sangue de pessoas residentes próximas a áreas de risco de intoxicação tenham reduzido 
drasticamente nas últimas décadas, estudos recentes sugerem que, mesmo em concentrações inferiores a $10 \mu \mathrm{g} / \mathrm{dl}^{1}$, a contaminação pode afetar certos aspectos do desenvolvimento infantil, incluindo a aprendizagem e o repertório comportamental (Canfield, Henderson, Cory-Slechta, Cox, Jusko, \& Lanphear, 2003; Chandramouli, Steer, Ellis, \& Emond, 2009; Chiodo, Jacobson, \& Jacobson, 2004; Dascanio et al., 2015; Needleman \& Gatsonis, 1990).

Os efeitos da intoxicação por chumbo com níveis acima de $10 \mu \mathrm{g} / \mathrm{dl}$ foram alvo de inúmeros estudos e estão associados com alterações neurocomportamentais em crianças, tais como déficits na linguagem e na cognição e problemas de hiperatividade (Dascanio et al., 2012; Kaufman, 2001; Kordas et al., 2006). Schwartz (2004) e Plusquellec et al. (2010) apresentaram resultados considerados conclusivos quanto aos danos definitivos causados pelo chumbo no nível intelectual, em termos de déficit cognitivo, principalmente em crianças. Adicionalmente, desde a década de 1980, quando se iniciaram os estudos mais sistemáticos sobre contaminação por chumbo e repertório social, os pesquisadores deste campo encontraram forte associação com problemas de conduta, tais como: agressividade, impulsividade, problemas de comportamento externalizante e internalizante (Braun et al., 2008; Fraser, Muckle, \& Després, 2006; Marcus, Fulton, \& Clarke, 2010; Needleman, McFarland, Ness, Fienberg, \& Tobin, 2003; Olympio et al. 2010; Wright et al., 2008).

Embora bastante documentado, é difícil definir os efeitos diretos da contaminação por chumbo, em razão da interação entre o nível de chumbo e inúmeras variáveis como: nível socioeconômico; variáveis individuais, como sexo, idade, temperamento; e práticas educativas. Sendo assim, todas essas possíveis variáveis moderadoras interagem de forma complexa com a concentração de chumbo para produzir diferentes desfechos sobre o desenvolvimento das crianças e adolescentes (Bellinger, Stilles, \& Needleman, 1992; Dascanio et al., 2015).

Os pesquisadores Rice e Barone (2000) sugeriram que a dificuldade na compreensão dos efeitos do chumbo no sangue está relacionada ao fato de que a maioria dos estudos focaliza domínios específicos do desenvolvimento, sem estudar os padrões de déficits que coexistem em crianças expostas desde o nascimento, por exemplo, em termos de déficits intelectuais e comportamentais (Bellinger, 2004). Este estudo caminha na direção dessa lacuna, buscando descrever a relação entre contaminação por chumbo e variáveis contextuais diversas, com foco nas relações entre plumbemia e desenvolvimento de comportamentos desejáveis, como as habilidades sociais, e indesejáveis, como os problemas de comportamento.

Para interagir satisfatoriamente com adultos e pares, em diferentes contextos, é necessário que as crianças adquiram um repertório de 
habilidades sociais culturalmente adaptadas, que incluem as habilidades de civilidade, cooperação, asserção, autocontrole, empatia, dentre outras (Del Prette, Del Prette, Gresham, \& Vance, 2012; Elliott \& Gresham, 2008). Ainda que não haja um consenso quanto a um sistema único de classificação de habilidades sociais relevantes na infância, as habilidades sociais têm sido definidas, em uma perspectiva comportamental, como o conjunto de comportamentos sociais do repertório de um indivíduo que favorece o relacionamento saudável e produtivo com as demais pessoas (Del Prette \& Del Prette, 2008). A presença de tais comportamentos tem alta probabilidade de maximizar reforçadores e minimizar estimulação aversiva para o sujeito (Del Prette \& Del Prette, 2010; Gresham, 2009).

Em estudo longitudinal com 294 crianças italianas, com idade média de 8,5 anos, Caprara, Barbaranelli, Pastorelli, Bandura e Zimbardo (2000) identificaram que o efeito positivo dos comportamentos prossociais (oferecer ajuda, compartilhar, ser gentil, cooperar) teve maior impacto sobre o desempenho acadêmico, cinco anos mais tarde, do que o efeito negativo dos problemas de comportamento, como a agressividade. Resultados semelhantes foram obtidos no Brasil, a partir de uma pesquisa com 16 estudantes que apresentavam dificuldades de leitura e escrita e que foram submetidos a dois tipos de intervenção (Molina \& Del Prette, 2006). Aqueles que receberam um treinamento de habilidades sociais apresentaram melhoras no repertório social e, adicionalmente, ganhos acadêmicos, enquanto que o grupo submetido a treinamento acadêmico melhorou apenas o desempenho acadêmico, sem ganhos no repertório social. Em um estudo realizado com 119 estudantes brasileiros de escolas públicas, comparando crianças com e sem problemas de aprendizagem, os autores Z. A. P. Del Prette, Del Prette, Gresham e Vance (2012) identificaram que a cooperação entre os pares foi o melhor preditor sociocomportamental do pertencimento da criança a um ou outro grupo. Esses resultados sugerem 0 papel das habilidades sociais como facilitadores acadêmicos, sinalizando que habilidades sociais específicas são importantes nesse processo.

Em contrapartida, os déficits nas habilidades sociais representam um fator de risco ao desenvolvimento infantil (Walker \& Severson, 2002), potencializando dificuldades acadêmicas e comportamentais. Em uma síntese dos estudos que relacionam habilidades sociais e problemas comportamentais, observou-se que as crianças com problemas de comportamento apresentaram: limitações no repertório de habilidades sociais e em competência social (Bandeira, Rocha, Souza, Del Prette, \& Del Prette, 2006; Sorlie, Hagen, \& Ogden, 2008); acadêmica (Cia \& Barham, 2009; D'Abreu \& Marturano, 2010; Molina \& Del Prette, 2006); indicadores de status sociométrico negativo, 
como possuir menos amigos (Gresham, MacMillan, Bocian, Ward, \& Forness, 1998); maior grau de solidão (Gresham, Lane, MacMillan, \& Bocian, 1999; Weeks, Coplan, \& Kingsbury, 2009); além de sofrerem vitimização por parte dos pares (Perren \& Alsaker, 2009).

É importante considerar que os fatores de risco e proteção ao desenvolvimento não são iguais em importância e que comprometimentos comportamentais e acadêmicos podem ser decorrentes de múltiplos fatores (Sameroff \& Seifer, 1990). Essa tese reforça a relevância de análises estatísticas capazes de identificar quais os indicadores dos fatores de risco e proteção. Os dados da literatura sugerem um conjunto de fatores de risco ao desenvolvimento (por exemplo: plumbemia; problemas de comportamento; déficits em habilidades sociais e de competência acadêmica; práticas educativas coercitivas; e baixo nível socioeconômico), cujo impacto parece envolver intrincada rede de interações que deve ser investigada mais intensivamente. É possível inferir, também, que um bom repertório de habilidades sociais reduz o impacto dos fatores de risco, funcionando como uma variável protetora, mediadora ou moderadora da relação entre aqueles fatores de risco e desfechos desfavoráveis ao desenvolvimento. Com base nesses estudos, relacionando plumbemia, habilidades sociais, desempenho intelectual, problemas de comportamento e práticas educativas parentais, confirma-se à relevância científica e social de melhor investigar esta temática.

Nesse estudo, considerando a situação de risco de crianças e adolescentes contaminadas por chumbo, buscou-se identificar as variáveis (habilidades sociais, práticas educativas, problemas de comportamento e competência acadêmica) que diferenciam as crianças e adolescentes com e sem intoxicação por chumbo e a possível função protetora das habilidades sociais sobre problemas de comportamento em ambos os grupos.

\section{Método}

O presente estudo foi aprovado pelo Comitê de Ética em Pesquisa (Processo no 2651/46/01/09, aprovado em 18/12/2009) e atendeu a todos os requisitos da Resolução no. 196, de 10 de outubro de 1996, que regulamenta a pesquisa com seres humanos.

\subsection{Participantes}

Participaram deste estudo 100 estudantes, dos quais 62\% de nível socioeconômico baixo (Classe $C$ ), segundo o instrumento Critério Brasil (ABEP, 2010), com e sem intoxicação ${ }^{2}$ pelo metal chumbo. As mães e os professores também participaram como informantes. A 
idade dos estudantes variava de 8 a 17 anos $(M=13,12 ; D P=2,47)$, sendo $54 \%$ meninas. Desses, 50 compuseram o grupo com plumbemia (GP) e residiam em uma cidade de aproximadamente 340 mil habitantes situada no interior do Estado de São Paulo, em um bairro residencial contaminado por chumbo a partir da exposição de resíduos tóxicos pelas chaminés de uma fábrica de baterias, cuja interdição ocorreu no ano de 2002. Os outros 50 participantes compuseram o grupo de comparação, sem plumbemia (GC), e residiam em uma cidade vizinha, com aproximadamente 50 mil habitantes, sem histórico ou indicativo de contaminação pelo metal chumbo, como residir próximo a tráfego urbano ou a indústrias de baterias. A equivalência entre os grupos GP e GC foi confirmada por meio do teste do Qui-quadrado para gênero $\left(X^{2}=0,667 ; p=0,41\right)$; série escolar $\left(X^{2}=345,50 ; p=0,73\right)$ e nível socioeconômico $\left(X^{2}=\right.$ $363,50 ; p=0,98)$. Com 0 teste $t$ de Student confirmou-se a equivalência para a idade $(t=-1,61, p=0,87)$.

\subsection{Instrumentos de medida}

Sistema de Avaliação de Habilidades Sociais (SSRS-BR). Instrumento de relato de habilidades sociais, comportamentos problemáticos e competência acadêmica, produzido por Gresham e Elliott (1990), com tradução e validação semântica para o português (Bandeira, Del Prette, Del Prette, \& Magalhães, 2009). A versão para professores foi utilizada conforme a validação para a população brasileira, pois apresentou consistência interna satisfatória para este estudo, com alfa total de 0,88 e nos componentes: (F1) Responsabilidade, $a=$ 0,92; (F2) Assertividade, $a=0,86$; (F3) Autocontrole, $a=0,88$; (F4) Autodefesa, $a=0,79$; e (F5) Cooperação, $a=0,78$. Para a escala total de problemas de comportamento o valor de alfa foi de 0,93; para problemas de comportamento externalizante, $a=0,94 \mathrm{e}$ internalizante, $a=0,87$; para competência acadêmica o valor do alfa foi de 0,97 . Na versão das habilidades sociais para as crianças, a análise da consistência interna pelo Alfa de Cronbach indicou baixa fidedignidade, pelo que se procedeu a Análise Exploratória em Componentes Principais que apresentou uma estrutura em quatro componentes, explicando $42 \%$ da variância: (F1) - designado "Expressão de sentimento" com $a=0,78 ;(\mathrm{F} 2)$ - "Responsabilidade", $a=0,70$; (F3) - "Assertividade", $a=0,69$; e (F4) - "Civilidade", $a=$ 0,60 (ver Dascanio, 2012). A escala total foi composta por 23 itens, com alfa total de 0,80 , sendo considerada consistente, visto o valor de alfa em cada componente. Neste estudo, utilizou-se o escore total de habilidades sociais e a classe autocontrole avaliados pelo professor e, também, o escore total de habilidades sociais e as classes assertividade e civilidade autoavaliadas e as escalas de problemas de comportamento e competência acadêmica ${ }^{3}$. 
Inventário de Habilidades Sociais para Adolescentes (IHSA-DelPrette, Del Prette \& Del Prette, 2009). Instrumento de autorrelato para avaliação de habilidades sociais, constituído por 38 itens, que contemplam as principais demandas de desempenho interpessoal de adolescentes entre 12 e 17 anos, junto a diferentes interlocutores e contextos. Essa escala é subdivida em seis fatores, que reúnem habilidades sociais de: (F1) Empatia; (F2) Autocontrole; (F3) Civilidade; (F4) Assertividade; (F5) Abordagem Afetiva e (F6) Desenvoltura Social. Para cada um dos 38 itens, o adolescente deve estimar: (a) quão difícil é para ele apresentar a reação indicada no item e (b) qual a frequência com que apresenta a reação indicada em cada item. Nesses dois indicadores (frequência e dificuldade), as respostas são mensuradas em uma escala do tipo Likert de cinco pontos de acordo com a frequência estimada de ocorrência ( 0 a 2 vezes; 3 a 4 vezes; 5 a 6 vezes; 7 a 8 vezes e 9 a 10). Neste estudo, utilizou-se apenas o indicador de frequência e sua avaliação da consistência interna, indicou valores de alfa satisfatórios ( $a=0,93$ para a escala total e de 0,69 a 0,82 para as subescalas). Para viabilizar a correspondência com a escala SSRS-BR (usada na autoavaliação das crianças), a análise restringiu-se ao escore total e às subescalas de assertividade e civilidade, comuns aos dois inventários.

Inventário de Estilos Parentais (IEP-Gomide, 2006). Instrumento de relato que objetiva avaliar as práticas educativas parentais e fornecer o índice de estilo parental. Compõe-se por 42 itens, que são respondidos em uma escala do tipo Likert de acordo com a frequência estimada de ocorrência (Nunca - 0 a 2 vezes, Às vezes - 3 a 7 vezes, Sempre -8 a 10 vezes). O IEP pode ser respondido pelos pais, informando as práticas educativas que adotam em relação ao filho, e pelos filhos, informando as práticas educativas utilizadas por seus pais. As questões são basicamente as mesmas e adaptadas ao tipo de respondente. A estrutura fatorial original do IEP agrupa os itens em sete subescalas de práticas educativas, cada uma delas com seis itens, sendo cinco práticas caracterizadas como negativas (negligência, abuso físico, disciplina relaxada, punição inconsistente e monitoria negativa) e duas como positivas (monitoria positiva e comportamento moral). Para esta pesquisa, foi utilizada uma estrutura fatorial simplificada, produzida no estudo de Dascanio (2012), com três componentes similares, tanto para a versão pais como para a versão filhos (Prática Indutiva, Prática Inconsistente e Prática Negligente), que se mostrou mais consistente, explicando $40,59 \%$ e 32,07\%, respectivamente, da variância dos dados.

\subsection{Procedimento de coleta de dados}


A avaliação dos estudantes foi realizada nas escolas, com autorização prévia da direção e dos pais, ocorreu de forma individual, em sala cedida pela direção. O instrumento SSRS-BR foi respondido pelas crianças na faixa etária de 8 a 12 anos. O IHSA-Del-Prette foi respondido pelos adolescentes de 13 a 17 anos. O IEP, na versão filhos, foi respondido tanto pelas crianças quanto pelos adolescentes. O SSRS-BR, versão professor, foi respondido pelo professor $(N=15)$ também na própria escola, individualmente.

\subsection{Procedimentos éticos}

A assinatura do Termo de Consentimento Livre e Esclarecido (TCLE), o preenchimento dos itens do Critério Brasil (para avaliação socioeconômica) e do IEP foram realizados pelas mães dos participantes na residência. Tanto para as mães como para as crianças e adolescentes, o IEP foi aplicado em forma de entrevista de modo a garantir a compreensão pelos participantes (Processo $n^{\circ}$ 2651/46/01/09, aprovado em 18/12/2009).

\subsection{Análise dos dados}

As análises dos dados foram efetuadas com o programa PASW-18 for Windows. Para a avaliação das habilidades sociais, como foram utilizados dois instrumentos, um para cada faixa etária, procedeu-se à conversão do escore bruto do teste em valores lineares de $Z$ e, posteriormente, em valores de $T$, de modo a permitir a comparação, sob mesma métrica, entre crianças e adolescentes. Em virtude da baixa correspondência entre as classes de habilidades sociais mensuradas pelas escalas SSRS-BR, na versão criança, e IHSA-DelPrette, foram utilizados apenas os escores total de habilidades sociais e a subescala assertividade, todos convertidos em valores de $T$, conforme recomendado por Tabachnick e Fidell (2001).

Em seguida, procedeu-se a Análise de Variância de um fator (ANOVA one-way), para examinar possíveis diferenças nos grupos de plumbemia para as variáveis investigadas. Apesar desta investigação contar com apenas dois grupos (GP x GC) foi utilizada a ANOVA, uma vez que as variáveis analisadas apresentaram distribuição normal e com variâncias populacionais homogêneas, justificando-se sua utilização para obter analises mais robustas. A partir dos resultados da ANOVA, procedeu-se às análises discriminantes para testar quais variáveis de critério melhor diferenciavam os participantes.

Além disso, foi testada a relação de mediação entre algumas variáveis. A mediação é caracterizada quando a relação entre uma variável independente e uma determinada variável dependente é com frequência mediada por uma terceira variável, que "transporta" o 
efeito da variável independente sobre a dependente (Marôco, 2010). Para testar a função mediadora de uma variável, é necessário preencher as seguintes condições: (1) relação significativa entre a variável independente e mediadora na primeira equação; (2) variável mediadora com efeito significativo sobre a independente na segunda equação; (3) redução da importância da variável independente ao modelo com a adição da variável mediadora. A mediação na interação é confirmada quando, na terceira equação, a variável independente afeta menos e a mediadora afeta mais a variável dependente (Marôco, 2010).

As análises de mediação foram realizadas por meio de regressões lineares simples, verificando-se a significância estatística das relações expressas nos pontos (1) e (3), descritas anteriormente. O efeito da mediação é confirmado se os resultados dos testes Sobel e Aroian ${ }^{4}$, for significativo $(Z>1,96 ; p<0,05)$, conforme sugerido por Marôco, (2010).

\section{Resultados}

Os resultados são apresentados, inicialmente, considerando as análises de comparação entre os grupos, por meio da análise de variância (ANOVA de um fator), posteriormente apresentam-se as variáveis de diferenciação entre os grupos, com a análise discriminante e, em seguida, a análise de mediação entre as habilidades sociais e problemas de comportamento.

Pela Tabela 1 apresenta-se a comparação entre os grupos com plumbemia (GP) e sem plumbemia (GC) no tocante as habilidades sociais, problemas de comportamento, competência acadêmica e práticas educativas. 
Tabela 1

Dados descritivos da Média (M) e Desvio Padrão (DP) das habilidades sociais, problemas de comportamento, competência acadêmica e práticas educativas parentais

\begin{tabular}{|c|c|c|c|c|}
\hline Versão & Variáveis & $\begin{array}{c}G P \\
M(D P)\end{array}$ & $\begin{array}{c}G C \\
M(D P)\end{array}$ & $p$ \\
\hline \multirow{8}{*}{ Professor } & Responsabilidade & $17,94(5,78)$ & $21,60(7,23)$ & $0,006^{* *}$ \\
\hline & Assertividade & $9,56(3,61)$ & $12,26(4,48)$ & 0,392 \\
\hline & Autocontrole & $10,30(3,07)$ & $12,12(4,28)$ & $0,017^{*}$ \\
\hline & Autodefesa & $2,80(1,44)$ & $3,40(1,90)$ & 0,079 \\
\hline & Cooperação & $3,64(1,55)$ & $3,66(2,32)$ & 0,960 \\
\hline & $\begin{array}{l}\text { PC } \\
\text { externalizante }\end{array}$ & $8,32(4,44)$ & $2,74(4,60)$ & $0,000 * *$ \\
\hline & PC internalizante & $4,60(2,81)$ & $1,48(2,24)$ & $0,000 * *$ \\
\hline & $\begin{array}{l}\text { Competência } \\
\text { acadêmica }\end{array}$ & $28,22(6,80)$ & $31,86(10,15)$ & $0,038^{*}$ \\
\hline \multirow[t]{2}{*}{ Estudante } & HS Global & $51,86(9,89)$ & $49,38(9,22)$ & 0,199 \\
\hline & Assertividade & $53,37(8,40)$ & $47,70(9,77)$ & $0,002^{*}$ \\
\hline \multirow{3}{*}{ Mãe } & Indutiva & $15,70(3,51)$ & $14,86(3,19)$ & 0,222 \\
\hline & Negligência & $8,76(4,13)$ & $8,68(3,11)$ & 0,913 \\
\hline & Coercitiva & $4,90(3,13)$ & $4,88(2,42)$ & 0,972 \\
\hline \multirow{3}{*}{ Estudante } & Indutiva & $13,44(3,47)$ & $14,86(3,27)$ & $0,038^{*}$ \\
\hline & Inconsistente & $8,80(3,53)$ & $6,74(2,82)$ & $0,002^{*}$ \\
\hline & Coercitiva & $6,32(3,94) a$ & $6,92(4,05) \mathrm{a}$ & 0,455 \\
\hline
\end{tabular}

Nota. ${ }^{* *} p<0,001 ;{ }^{*} p<0,05$

Para as habilidades sociais avaliadas pelo professor, constatou-se diferença entre os grupos apenas para as habilidades sociais: responsabilidade e autocontrole. Para essas habilidades verificaramse melhor repertório para o grupo de comparação em relação ao grupo de plumbemia $(F=7,81 ; p=0,06$ e $F=5,95 ; p=0,017$, respectivamente). Para as outras classes de habilidades sociais: assertividade, autodefesa e cooperação não foram detectadas diferenças significativas entre os grupos.

Observou-se também uma diferença significativa entre os dois grupos para as variáveis: problemas de comportamento externalizante $(F=$ 
$38,70 p<0,001)$, internalizante $(F=37,71 ; p<0,001)$ e competência acadêmica $(F=4,44 ; p=0,038)$, indicando maior freqüência de problemas de comportamento e menor competência acadêmica para o grupo de plumbemia.

Ao considerar a autoavaliação dos estudantes, detectou-se diferença significativa para a habilidade social da classe assertividade $(F=$ 9,66; $p=0,002)$, em favor dos participantes com plumbemia, divergindo da avaliação do professor que não encontrou diferença para esta classe. Considerando o escore global de habilidades sociais, não foi identificada diferença entre os grupos.

No tocante às práticas educativas avaliadas pelas mães, não houve diferença estatisticamente significativa entre os grupos, já na autoavaliação dos filhos foi detectada diferença para as práticas educativas indutiva $(F=4,43 ; p=0,038)$, em favor do grupo de comparação e, para as práticas educativas inconsistente $(F=10,36$; $p=0,002)$, indicando que as práticas utilizadas pelas mães do grupo de plumbemia são mais inconsistentes quando comparadas ao grupo sem plumbemia.

Neste estudo buscou-se, também, identificar, por meio da análise discriminante, qual a importância relativa das variáveis apontadas pela ANOVA como significativas, a fim de diferenciar as crianças com intoxicação por chumbo daquelas sem intoxicação.

$\mathrm{Na}$ Tabela 2 encontram-se as variáveis que são mais relevantes na capacidade discriminativa dos grupos.

Tabela 2

Matriz estrutural das variáveis utilizadas na análise discriminante

\begin{tabular}{|c|c|c|}
\hline Preditores & $\begin{array}{c}\text { Função } \\
1\end{array}$ & $\begin{array}{c}\text { Coeficiente de } \\
\text { correlação canônica } \\
\text { (c.c.)* }\end{array}$ \\
\hline Responsabilidade & 0,524 & $-0,255$ \\
\hline Autocontrole & 0,218 & $-0,223$ \\
\hline Autodefesa & $-0,072$ & $-0,162$ \\
\hline $\begin{array}{l}\text { Problema de comportamento } \\
\text { externalizante }\end{array}$ & 0,984 & $0,563^{*}$ \\
\hline $\begin{array}{l}\text { Problema de comportamento } \\
\text { internalizante }\end{array}$ & 0,643 & $0,561^{*}$ \\
\hline Competência acadêmica & $-0,016$ & $-0,192$ \\
\hline Assertividade & 0,385 & 0,284 \\
\hline Prática Parental Indutiva & $-0,271$ & $-0,192$ \\
\hline Pratica Parental Inconsistente & 0,310 & 0,294 \\
\hline
\end{tabular}

As variáveis constantes na Tabela 2 representam uma função discriminante significativa (Wilks'Lambda $=0,450 ; \mathrm{X}^{2}=74,72 ; p<$ 0.0001). Os coeficientes de correlação canônicos (c.c.) obtidos mostraram que os aspectos que mais diferenciavam os participantes 
do seu grupo de referência, em ordem decrescente, foram os problemas de comportamento externalizante $($ c.c. $=0,563)$ e internalizante (c.c. $=0,561$ ).

A Tabela 3 apresenta a classificação dos grupos por meio da análise discriminante.

Tabela 3

Resultados da classificação e funcões classificatórias usadas

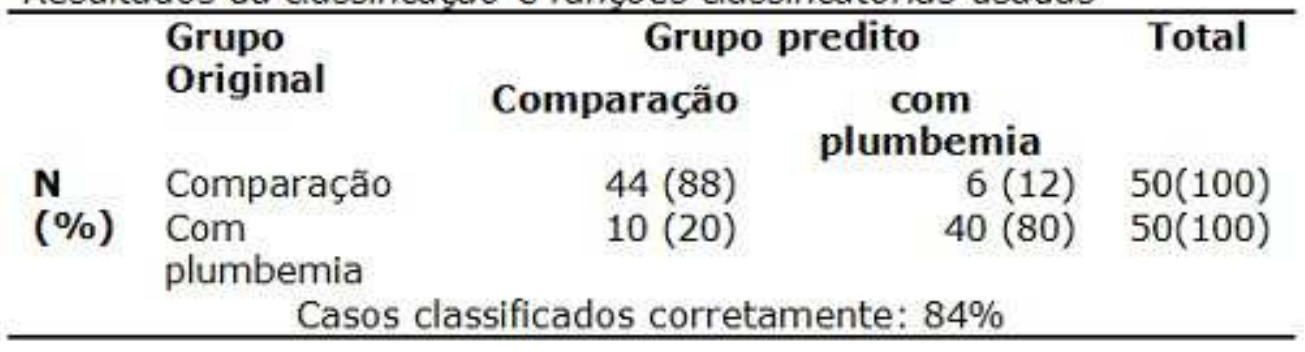

Pela Tabela 3 tem-se que a percentagem de participantes classificados corretamente com a classificação original foi de $84 \%$. Observa-se que para o grupo de comparação, sem plumbemia, $88 \%$ dos participantes foram classificados corretamente e $12 \%$ foram classificados com pertencentes ao grupo com plumbemia. Já para os participantes do grupo com plumbemia, a classificação correta representou $80 \%$ dos participantes e a incorreta $20 \%$.

A Tabela 4 mostra as análises de mediação entre as habilidades sociais, plumbemia e problemas de comportamento. Vale ressaltar que, para essas análises, optou-se por utilizar o escore total de habilidades sociais avaliados pelo professor, visto que o mesmo é considerado pela literatura como um bom avaliador do repertório social infantil (Feitosa, Del Prette, \& Loureiro, 2007).

Tabela 4

Análise da função mediadora das habilidades sociais (HS) entre as variáveis plumbemia ( $P b-S)$ e problemas de comportamento $(P C)$

\begin{tabular}{|c|c|c|c|c|c|c|}
\hline \multicolumn{2}{|c|}{$\begin{array}{c}\text { Equações de } \\
\text { Mediação }\end{array}$} & \multirow{2}{*}{$\begin{array}{c}\text { B } \\
-5.880\end{array}$} & \multirow{2}{*}{$\begin{array}{c}\text { SE } \\
2.316\end{array}$} & \multirow{2}{*}{$\begin{array}{c}\boldsymbol{\beta} \\
-.248\end{array}$} & \multirow{2}{*}{$\begin{array}{c}\boldsymbol{p} \\
.013\end{array}$} & \multirow{2}{*}{$\begin{array}{c}\boldsymbol{R}^{2} \\
.052\end{array}$} \\
\hline $\begin{array}{l}\text { Primeira } \\
\text { VI } \times M\end{array}$ & $\mathrm{~Pb}-\mathrm{S} \times \mathrm{HS}$ & & & & & \\
\hline $\begin{array}{l}\text { Segunda } \\
M \times V D\end{array}$ & $\mathrm{HS} \times \mathrm{PC}$ & -1.247 & .129 & -.699 & .000 & .483 \\
\hline \multirow[t]{2}{*}{$\begin{array}{l}\text { Terceira } \\
\text { VI.M } \times \text { VD }\end{array}$} & $\begin{array}{c}\mathrm{Pb}-\mathrm{S} \times \\
\mathrm{PC}\end{array}$ & 6.371 & .756 & .480 & .000 & .699 \\
\hline & $\mathrm{HS} \times \mathrm{PC}$ & -.325 & .032 & -.579 & .000 & \\
\hline
\end{tabular}

É possível perceber que as relações entre as variáveis são significativas tanto na primeira quanto na segunda equação. Já na terceira, é possível notar que a plumbemia, por meio da habilidade 
social, explicou $70 \%$ da variância dos problemas de comportamento, enquanto que sozinhas, a plumbemia explicou $5 \%$ e as habilidades sociais $48 \%$. Aplicado o teste Sobel e o de Aroian para verificar a confiabilidade desse resultado, observou-se um índice aceitável para 0 efeito de mediação $(Z=2.544 ; p=0.0109$ e $Z=-2.544 ; p=$ 0,0109 , respectivamente).

\section{Discussão e conclusões}

Os resultados encontrados mostraram que os estudantes do grupo de comparação (sem plumbemia) apresentaram maior repertório de habilidades sociais das classes responsabilidade (compromisso com as tarefas e com as pessoas no ambiente escolar e seguir instruções) e autocontrole (reagir de forma apropriada à pressão, gozação ou provocações dos colegas e negociar situações de conflito) do que aqueles estudantes que foram intoxicadas por chumbo (GP), considerando a avaliação do repertório social feita pelo professor. Por outro lado, esses últimos apresentaram mais problemas de comportamento externalizante e internalizante, o que se alinha aos estudos que salientam comprometimentos para os indivíduos intoxicados. Tais resultados vão ao encontro de todos os estudos pesquisados, cuja associação entre plumbemia e repertório social é negativa, tendo o professor como avaliador (Marcus, Fulton, \& Clarke, 2010; Needleman et al., 2003; Olympio et al. 2010; Wright et al. 2008).

A competência acadêmica também foi avaliada pelo professor, indicando maior competência para os estudantes do grupo sem plumbemia quando comparado aos estudantes com plumbemia. Os estudos da área de toxicologia reconhecem o comprometimento da capacidade intelectual de indivíduos intoxicados por chumbo (Needleman \& Gatsonis, 1990; Needleman et al., 2003; Tong, Mcmichael, \& Baghurst, 2000). É importante considerar também possibilidade de o professor, ao avaliar o comportamento da criança como socialmente habilidoso, generalizar sua avaliação mais favorável também para a competência acadêmica dessa criança (Feitosa, Del Prette, \& Loureiro, 2007), em termos de efeito "halo".

Ao considerar a avaliação do próprio estudante em relação ao seu repertório de habilidades sociais, encontrou-se que os estudantes com plumbemia relataram ser mais assertivos do que aqueles sem plumbemia, divergindo da avaliação do professor. Esse resultado contraria todos os estudos pesquisados sobre a relação entre plumbemia e repertório social (Marcus, Fulton, \& Clarke, 2010; Needleman, et al. 2003; Tong, Mcmichael, \& Baghurst, 2000) e deve ser tomado com bastante cautela. Em estudos anteriores com esses mesmos participantes levantou-se a hipótese de que a relação 
positiva entre plumbemia e assertividade poderia estar relacionada ao fato dessas crianças terem recebido uma atenção diferenciada dos órgãos de saúde da região, o que pode ter caracterizado um treino indireto, que gerou a aprendizagem incidental de habilidades assertivas (Dascanio, 2012; Dascanio et al. 2015). No período de 2002 a 2006, quando houve a descoberta da intoxicação por chumbo na região, as crianças e adolescentes com plumbemia eram bebês e crianças, respectivamente quando foram alvos de intensiva assistência multiprofissional junto à Clínica Escola de um Centro de Psicologia Aplicada, o que não ocorreu com os participantes sem plumbemia. Nesse período foram requeridas a responder perguntas sobre como se sentiam, por meio de uma anamnese, a autoavaliar suas condições gerais de funcionamento psicológico, a descrever rotinas, cumprimentar pessoas, entre outros aspectos. (Dascanio, Rodrigues, \& Valle, 2010). Essas demandas podem ter constituído contingências na história de vida dessas crianças, que as ajudaram a desenvolver comportamentos mais assertivos, como os de expressar sentimentos negativos, falar sobre as próprias qualidades ou defeitos, recusar pedidos, entre outros (Del Prette \& Del Prette, 2009). Destaca-se que não se pretende atribuir o maior repertório das crianças plumbímicas à avaliação mulitprofissional, porém, esta foi uma condição diferenciada em relação aos outros estudos da área e merece ser melhor investigada.

Estudos futuros poderiam investigar melhor a divergência de resultados em relação habilidade social da classe assertividade na avaliação do professor e dos próprios estudantes, pois estudos apontam que, normalmente, há convergência entre esses avaliadores: professores e estudantes (Gresham, Elliot, Cook, Vance, \& Kettler, 2010; Renk \& Phares, 2004).

Em relação às práticas educativas parentais, apenas quando o avaliador foi o filho, identificou-se diferença entre os grupos. Os estudantes intoxicados por chumbo avaliaram que seus pais utilizamse menos de práticas parentais indutivas e mais de práticas inconsistentes do que a avaliação dos filhos do grupo de comparação em relação a seus pais. Esse resultado concorda com a literatura se tomarmos como parâmetro apenas a avaliação das habilidades sociais feita pelo professor, visto os estudos que sinalizam a relação positiva entre as práticas parentais positivas (Gomide, 2003) e/ou indutivas com um repertório elaborado de habilidades sociais e com um bom relacionamento com pares e com a família (Dishion \& McMahon, 1998). Já o fato dos estudantes intoxicados relatarem maior uso de práticas parentais inconsistentes pode relacionar-se com a presença de problemas de comportamento em seu repertório, ou seja, por apresentarem mais problemas de comportamento do que as crianças não intoxicadas, suscitam práticas parentais mais consistentes, porém, esses pais, não conseguem suprir essa possível necessidade 
diferenciada dos filhos, alternando entre práticas educativas positivas e negativas.

Os resultados de análise discriminante permitiram classificar corretamente $84 \%$ dos casos, mostrando que os aspectos que mais diferenciaram os estudantes com plumbemia daqueles sem foram os problemas de comportamento externalizante e internalizante. Nesta perspectiva, o estudo confirma uma associação entre estar intoxicado por chumbo e a emissão de comportamentos-problema. Esse resultado é coerente com estudos considerados conclusivos sobre a relação entre plumbemia e problemas de comportamento externalizante, internalizante e criminalidade (Bellinger, Leviton, Allred, \& Rabinowitz, 1994; Chen, Cai, Dietrich, Radcliffe, \& Rogan, 2007; Wright, et al. 2008).

No tocante à análise da mediação, observa-se o papel das habilidades sociais como mediadora da relação entre plumbemia e problemas de comportamento. Considerando que a plumbemia relaciona-se negativamente com os problemas de comportamento, as habilidades sociais parecem funcionar como um fator de proteção para mediar à relação entre plumbemia e problemas de comportamento, minimizando seu impacto no repertório social. Efetivamente, esses resultados sugerem que as habilidades sociais podem contribuir na proteção de crianças em situações de risco. É possível considerar que se o repertório de assertividade e habilidades sociais das crianças contaminadas por chumbo fosse equivalente ao das crianças do grupo de comparação, o impacto negativo da plumbemia sobre o repertório social poderia ter sido ainda maior, conforme descritos nos estudos que demonstraram a associação negativa entre essas variáveis (Chen et al., 2007; Needleman \& Gatsonis, 1990). Vale destacar que, conforme Sameroff e Seifer, (1990), nenhum fator de risco isolado pode ser considerado responsável por determinado comprometimento e que as crianças deste estudo estão expostas a vários fatores de risco, como baixo nível socioeconômico e práticas parentais inconsistentes, além da própria intoxicação por chumbo (Dascanio, Valle, \& Rodrigues, 2010).

Como descrito por Bellinger (2004) em revisão da literatura PSI, os efeitos diretos da intoxicação por chumbo são difíceis de identificar, em decorrência da coexistência de inúmeras variáveis. Fraser, Muckle e Després (2006) também sublinharam essa dificuldade, por meio de análises de mediação e moderação entre a exposição ao chumbo pré e pós-natal, a função motora e a impulsividade em crianças, sugerindo que os pesquisadores atuem no sentido de identificar os efeitos diretos e indiretos do chumbo sobre vários domínios do desenvolvimento infantil.

Considerando os limites da presente pesquisa (especialmente o tamanho reduzido da amostra), para estudos futuros, é preciso refletir sobre a avaliação das habilidades sociais e dos problemas de 
comportamento também pelos pais e por observação direta dos comportamentos, visto que, nessa investigação, encontraram-se resultados divergentes entre a autoavaliação e a avaliação do professor.

Em resumo, o conjunto dos resultados obtidos neste estudo reforça a ideia de que um repertório elaborado de habilidades sociais pode estar relacionado com a competência cognitiva e, por essa via, o sucesso acadêmico da criança.

\section{Referências}

Associação Brasileira de Empresas de Pesquisa (ABEP) (2010). Critério de classificação econômica Brasil. Recuperado em 22 de fevereiro, 2010, de: http://www.abep.org

Bandeira, M., Rocha, S. S., Souza, T. M. P., Del Prette, Z. A. P., \& Del Prette, A. (2006). Comportamentos problemáticos em estudantes do ensino fundamental: Características da ocorrência e relação com habilidades sociais e dificuldade de aprendizagem. Estudos em Psicologia, 11, 199-208.

Bandeira, M., Del Prette, Z. A. P., Del Prette, A., \& Magalhães, T. (2009). Validação das escalas de habilidades sociais, comportamentos problemáticos e competência acadêmica (SSRS-BR) no ensino fundamental. Psicologia: Teoria e Pesquisa, 25(2), 271-282. doi: 10.1590/S010237722009000200016

Bellinger, C. D., Stiles, M. K., \& Needleman, L. H. (1992). Low-level lead exposure, intelligence and academic achievement: a longterm follow-up study, Pediatrics, 90, 855-861.

Bellinger, D., Leviton, A., Allred, E., \& Rabinowitz, M. (1994). Preand postnatal lead exposure and behavior problems in schoolaged children. Environmental Research, 66, 12-30.

Bellinger, C. D. (2004). Lead, Pediatrics, 113, 1016-1022. doi: 10.1006/enrs.1994.1041

Braun, J. M., Froehlich, T. E., Daniels, J. L., Dietrich, K. N., Hornung, R., Auinger, P., \& Lanphear, B. P. (2008). Association of environmental toxicants and conduct disorder in U.S. Children: NHANES 2001-2004. Environmental Health Perspectives, 116, 956-962. doi: 10.1289/ehp.11177

Canfield, R. L., Henderson, C. R. Jr, Cory-Slechta, D. A., Cox, C., Jusko, T. A., \& Lanphear, B. P. (2003). Intellectual impairment in children with blood lead concentrations below $10 \mu \mathrm{g}$ per deciliter. New England Journal of Medicine, 348, 1517-1526. doi: 10.1056/NEJMoa022848

Caprara, G. V., Barbaranelli, C., Pastorelli, C. Bandura, A., \& Zimbardo, F. G. (2000). Prosocial foundations of children's 
academic achievement. Psychological Science, 11, 302-306. doi: $10.1111 / 1467-9280.00260$

Chandramouli, K., Steer, D. C., Ellis, M., \& Emond, A. M. (2009). Effects of early childhood lead exposure on academic performance and behaviour of school age children. Archives of Disease in Childhood, 94, 844-848. doi: 10.1136/adc.2008.149955

Chen, A., Cai, B., Dietrich, K. N., Radcliffe, J., \& Rogan, W. J. (2007). Lead exposure, IQ, and behavior in urban 5- to 7-year-olds: Does lead affect behavior only by lowering IQ? Pediatrics, 119, 650-658. doi: 10.1542/peds.2006-1973

Chiodo, L. M., Jacobson, S. W., \& Jacobson, J. L. (2004). Neurodevelopmental effects of postnatal lead exposure at very low levels. Neurotoxicology and Teratology, 26, 359-371. doi: $10.1016 /$ j.ntt.2004.01.010

Cia, F., \& Barham, E. J. (2009). Repertório de habilidades sociais, problemas de comportamento, autoconceito e desempenho acadêmico de crianças no início da escolarização. Estudos de Psicologia, 26, 45-55. doi: 10.1590/S0103166X2009000100005

D'Abreu, L. C. F., \& Marturano, E. M. (2010). Associação entre comportamentos externalizantes e baixo desempenho escolar: uma revisão de estudos prospectivos e longitudinais. Estudos de Psicologia, 15, 43-51. doi: 10.1590/S1413294X2010000100006

Dascanio, D. (2012). Habilidades sociais, plumbemia, funcionamento intelectual e variáveis sociodemográficas em crianças e adolescentes. Tese de Doutorado. Programa de Pós Graduação em Psicologia, Universidade Federal de São Carlos.

Dascanio, D., Rodrigues, O. M. P. R., \& Valle, T. G. M. (2010). Relação entre os estilos parentais e o desempenho intelectual de crianças com plumbemia. Avaliação Psicológica, 9(3), 461470. doi: S1677-04712010000300012

Dascanio, D., Olaz, O. F., Fontaine, A. M. G., Rodrigues, O. M. P. R., Del Prette, A., \& Del Prette, Z. A. P. (2012). Intellectual and academic performance of adolescents poisoned by lead: Relationship with social skills. Temas em Psicologia, 20(1), 4559. doi: $10216 / 70458 / 2 / 81434$

Dascanio, D., Del Prette, A., Barham, E. J., Rolim, R. P. M. O., Fontaine, V. G. M. A., \& Del Prette, Z. A. P. (2015.). Habilidades sociais, competência acadêmica e problemas de comportamento em crianças e adolescentes com diferentes níveis de plumbemia. Psicologia: Reflexão e Crítica, Porto Alegre, 28(1), 166-176. doi: 10.1590/1678-7153.201528118

Del Prette, Z. A. P., \& Del Prette, A. (2008). Um sistema de categorias de habilidades sociais educativas. Paidéia, Cadernos 
de Psicologia e Educação, 18(41), 517-530. doi: 10.1590/S0103-863X2008000300008

Del Prette, A., \& Del Prette, Z. A. P (2009). Inventário de Habilidades Sociais para Adolescentes (IHSA-Del-Prette). São Paulo: Casa do Psicólogo.

Del Prette, Z. A. P., \& Del Prette, A. (2010). Habilidades sociais e Análise do Comportamento: Proximidade histórica e atualidades. Perspectivas em Análise do Comportamento, 1(2), 38-49.

Del Prette, Z. A. P., Del Prette, A., Gresham, F. M., \& Vance, M. J. (2012). Role of social performance in predicting learning problems: Prediction of risk using logistic regression analysis. School Psychology International Journal, 2, 1-16. doi: $10.1177 / 0020715211430373$

Dishion, T. J., \& McMahon, R. J. (1998). Parental monitoring and the prevention of child and adolescent problem behavior: a conceptual and empirical formulation. Clinical Child and Family Psychology Review, 1, 61-75. doi: 10.1023/A:1021800432380

Elliott, S. N. \& Gresham, F. M. (2008). Social Skills Improvement system: Intervention Guide. Bloomington, MN: Pearson Assessments.

Feitosa, F. B., Del Prette, Z. A. P., \& Loureiro S. R. (2007). Acuracidade do professor na identificação de alunos com dificuldade de aprendizagem. Temas em Psicologia, 15(2), 237247. doi: S1413-389X2007000200008

Fraser, S., Muckle, G., \& Després, C. (2006). The relationship between lead exposure, motor function and behaviour in Inuit preschool children, Neurotoxicology and Teratology, 28, 18-27. doi: 10.1016/j.ntt.2005.10.008

Gresham, F. M. (2009). Análise do comportamento aplicada às habilidades sociais. In: Z. A. P. Del Prette, \& A. Del Prette (Orgs.). Psicologia das habilidades sociais: diversidade teórica e suas implicações (pp. 17-66). Petrópolis: Vozes.

Gresham, F. M. \& Elliott, S. N. (1990). Social Skills Rating System: Manual. Circle.

Gresham, F. M., MacMillan, D.L., Bocian, K. M., Ward, S. L. \& Forness, S.R. (1998). Comorbidity of Hyperactivity-impulsivityinattention and conduct problems: Risk factors in social, affective and academic domains. Journal of Abnormal Child Psychology, 26(5), 393-406.doi: 10.1023/A:1021908024028

Gresham, F.M., Lane, K.L., MacMillan, D.L. \& Bocian, K.M. (1999). Social and academic profiles of externalizing and internalizing groups: Risk factors for emotional and behavioral disorders. Behavioral Disorders, 24(3), 231-245.

Gresham, F. M., Elliott, S. N., Cook, C. R., Vance, M. J., \& Kettler, R. (2010). Cross-Informant Agreement for Ratings for Social Skill 
and Problem Behavior Ratings: An Investigation of the Social Skills Improvement System-Rating Scales, Psychological Assessment, 22, 157-166. doi: 10.1037/a0018124

Gomide, P. I. C. (2003). Estilos parentais e comportamento antissocial. In: A. Del Prette \& Z. A. P. Del Prette (orgs). Habilidades sociais, desenvolvimento e aprendizagem: Questões conceituais, avaliação e intervenção. Campinas: Alínea.

Gomide, P. I. C. (2006). Inventário de Estilos Parentais. Petrópolis: Vozes.

Kaufman, A. S. (2001). How dangerous are low (not moderat or hight) doses of lead for children's intellectual development. Archives of clinical Neuropsychology, 16(4), 403-431. doi: 10.1016/S0887-6177(00)00090-1

Kordas, K., Canfield, R. L., López, P., Rosado, J. L., Vargas, G. G., Cebrián, M. E., Stoltzfus, J. R. (2006). Deficits in cognitive function and achievement in Mexican first-graders with low blood lead concentrations. Environmental Research, 100, 371386. doi: 10.1016/j.envres.2005.07.007

Marcus, D. K., Fulton, J. J., \& Clarke, E. J. (2010). Lead and Conduct Problems: A meta-analysis. Journal of Clinical and Adolescent Psychology, 39, 234-241. doi: 10.1080/15374411003591455

Marôco, J. (2010). Análise Estatística com o PASW Statistics (exSPSS). Lisboa/Portugal: Report Number.

Molina, R. C. M., \& Del Prette, Z. A. P. (2006). Funcionalidade da relação entre habilidades sociais e dificuldades de aprendizagem. Psico - USF, 11, 53-63. doi: 10.1590/S141382712006000100007

Needleman, H. L., \& Gatsonis, C. A. C. (1990). Low-level lead exposure and the IQ of children: A meta-analysis of modern studies. The Journal of the American Medical Association, 263, 673-678. doi: 10.1001/jama.1990.03440050067035

Needleman, H. L., McFarland, C., Ness, R. B., Fienberg, S. E., \& Tobin, M. J. (2003). Bone lead levels in adjudicated delinquents. A case control study. Neurotoxicology and Teratology, 24, 711-717. doi: 10.1016/s0892-0362(02)00269-6

Olympio, K. P. R, Oliveira, P. V, Naozuka, J, Cardoso, M. R. A., Marques, A. F., Gunther, W. M. R., \& Bechara, E. J. H. (2010). Surface dental enamel lead levels and antisocial behavior in Brazilian adolescents. Neurotoxicology and Teratology, 32, 273279. doi: $10.1016 / \mathrm{j} . \mathrm{ntt} .2009 .12 .003$

Padula, N. A. M. R. e Grupo de Estudo e Pesquisa da Intoxicação por Chumbo em Crianças de Bauru et al. (2006). Intoxicação por chumbo e saúde infantil: Ações intersetoriais para 0 enfrentamento da questão. Cadernos de Saúde Pública, 22, 163-171. 
Perren, S., \& Alsaker, F. (2009). Depressive symptoms from kindergarten to early school age: Longitudinal associations with social skills deficits and peer victimization. Child and Adolescent Psychiatry and Mental Health, 3(28), 1-10. doi: 10.1186/17532000-3-28

Plusquellec, P., Muckle, G., Dewailly, E., Ayotte, P., Bégin, G., Desrosiers, C., Poitras, K. (2010). The relation of environmental contaminants exposure to behavioral indicators in Inuit preschoolers in Arctic Quebec. NeuroToxicology, 31, 17-25. doi: 10.1016/j.neuro.2009.10.008

Renk, K., \& Phares, V. (2004). Cross-informant ratings of social competence in children and adolescents. Clinical Psychology Review, 24, 239-254. doi: 10.1016/j.cpr.2004.01.004

Rice, D., \& Barone Jr., S. (2000). Critical periods of vulnerability for the developing nervous system: evidence from humans and animal models, Environmental Health Perspective, 108(3), 511533.

Sameroff, A. J., \& Seifer, R. (1990). Early contributors to developmental risk. In S. Weintraub (Ed.), Risk and protective factors in the development of psychopathology (pp. 52-66). New York: Cambridge University Press.

Schwartz, J. (2004). Societal benefits of reducing lead exposure. Environmental Research, 66, 105-124. doi: 10.1006/enrs.1994.1020

Sorlie, M. A., Hagen, K. A., \& Ogden, T. (2008). Social competence and antisocial behavior: Continuity and distinctiveness across early adolescence. Journal of Research on Adolescence, 18, 121-144. doi: 10.1111/j.1532-7795.2008.00553.x

Tabachnick, B. \& Fidell, L. (2001). Using multivariate statistics (4th edition). New York: Harper e Row.

Tong, S., Mcmichael, A. J. E., \& Baghurst, P. A. (2000). Interactions between environmental lead exposure and sociodemographic factors on cognitive development. Archives of Environmental Health, 55, 330-335. doi: 10.1080/00039890009604025

Walker, H. M., \& Severson, H. H. (2002). Developmental prevention of at-risk outcomes for vulnerable antisocial children and youth. In K. L. Lane, F. M. Gresham \& T. E. O'Shaughnessy (Orgs.). Children with or at risk for emotional and behavioral disorders (177-194). Boston: Allyn \& Bacon.

Weeks, M., Coplan, R. J., \& Kingsbury, A. (2009). The correlates and consequences of early appearing social anxiety in young children. Journal of Anxiety Disorders, 23(7), 965-972.

Wright, P. J., Dietrich, K. N., Douglas Ris, M., Hornung, R. W., Wessel, S. D., Lanphear, B. P., Ho, M., \& Rae, M. N. (2008). Association of Prenatal and Childhood Blood Lead 


\section{Concentrations with Criminal Arrests in Early Adulthood. Medicine, 5(5), 732-740. doi: 10.1371/journal.pmed.0050101}

\section{Endereço para correspondência Denise Dascanio}

Universidade Paulista (Campus de Bauru)

Departamento de Ciências Humanas

Rua Luís Levorato, 2-140, Chácaras Bauruenses, CEP 17048-290, Bauru - SP, Brasil Endereço eletrônico: denisedascanio@yahoo.com.br

\section{Zilda Aparecida Pereira Del Prette}

Universidade Federal de São Carlos

Departamento de Psicologia

Rod. Washington Luís, Km 235, s/n, Monjolinho, CEP 13565-905, São Carlos - SP, Brasil

Endereço eletrônico: zdprette@ufscar.br

\section{Anne Marie Germane Victorine Fontaine}

Universidade do Porto (Portugal)

Faculdade de Psicologia e de C. da Educação

Centro de Psicologia da Universidade do Porto

Rua Alfredo Allen, 4200-135, Porto, Portugal

Endereço eletrônico: fontaine@fpce.up.pt

Recebido em: 06/03/2015

Reformulado em: 18/05/2015

Aceito para publicação em: 19/05/2015

\section{Notas}

* Doutora em Psicologia pela Universidade Federal de São Carlos (UFSCar). Docente da Universidade Paulista (UNIP/Bauru). Faz parte do grupo de pesquisa Relacionamento Interpessoal e Habilidades Sociais (www.rihs.ufscar.br).

** Doutora em Psicologia, com pós-doutorado no exterior em Habilidades Sociais, professora titular, vinculada ao Departamento de Psicologia e ao Programa de PósGraduação em Psicologia da UFSCar, bolsista de Produtividade em Pesquisa do CNPq (Pq-1A), coordenadora do Grupo Relações Interpessoais e Habilidades Sociais (http://www.rihs.ufscar.br), publicou, em coautoria com Almir Del Prette, artigos, livros e inventários de avaliação em Habilidades Sociais.

*** Doutora em Psicologia, professora catedrática da Faculdade de Psicologia e de Ciências da Educação da U. do Porto (Portugal); membro do Centro de Psicologia da U. do Porto (CPUP), no qual coordena a linha Cultura, Normatividade e Diversidade. Publicou numerosos livros e artigos em revistas nacionais e internacionais. É actualmente coordenadora do projecto Europeu INTERFASOL - (Intergenerational Family Solidarity across Europe: www.cost.eu COST IS1311).

${ }^{1}$ Órgãos de regulamentação internacional como a Organização Mundial da Saúde (OMS), O CDC e a American Conference of Governmental Industrial Hygienists (ACGIH) estabelecem como aceitável uma concentração de chumbo no sangue das crianças menor que $10 \mu \mathrm{g} / \mathrm{dl}$.

${ }^{2}$ O diagnóstico da plumbemia foi realizado em 2002 e 2006, a partir da técnica de espectrometria de absorção atômica, por forno de grafite, com corretor Zeeman, modelo SIMAA 6000 Perkin Elmer. Foram consideradas intoxicadas crianças com plumbemia acima de $10 \mu \mathrm{g} / \mathrm{dl}$ (Padula, 2006).

3 Para selecionar as variáveis que compuseram as análises de regressão linear, inicialmente, procedeu-se às análises de correlações (Pearson) para verificar quais se correlacionavam com as dependentes. A seleção das variáveis obtidas com o 
SSRS, versão criança, baseou-se também na correspondência entre as variáveis do instrumento IHSA-Del-Prette.

${ }^{4}$ Disponíveis em: http://quantpsy.org/sobel/sobel.htm 